\title{
Occult Foreign Bodies in ENT
}

\author{
Bijan Kumar Adhikary, ${ }^{1}$ Subhra Deb Biswas, ${ }^{2}$ Avick Das, ${ }^{2}$ Swapan Kumar Ghosh ${ }^{2}$
}

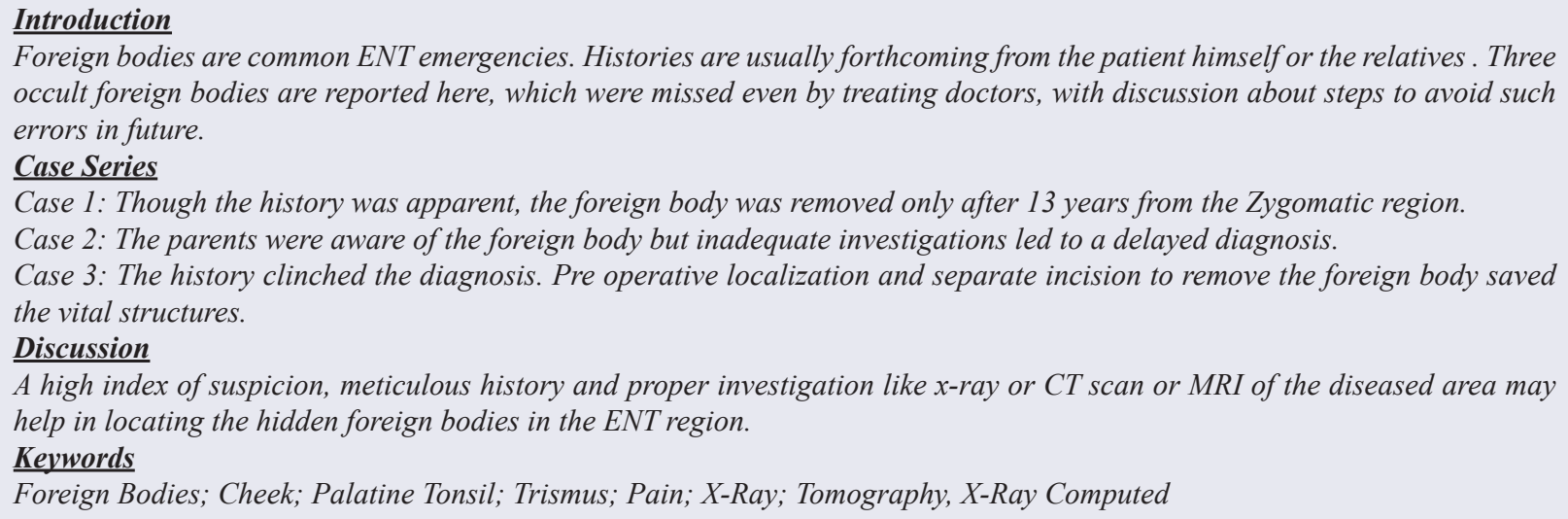

$\mathrm{F}$ oreign bodies in natural orifices and aerodigestive tract are common. A foreign body in ear, nose or throat can cause harm by its mere presence if immediate medical attention is not sought. ${ }^{1}$ Penetrating foreign bodies may be difficult to diagnose in absence of definite history. Here we present three case reports of penetrating foreign bodies those were missed for considerable time before being diagnosed by appropriate radiological investigations.

\section{Case Series}

Case 1

A 38 year old military personnel presented to the OPD with the complaint of a swelling over his right cheek for the past 7 months. There was a $2 \mathrm{~cm} \times 2 \mathrm{~cm}$ cystic swelling tender on palpation, located below the zygoma. There was a scar over right cheek, which he informed, was due to trauma from a blast injury while serving in Kashmir 13 years back. The wound was treated at local field hospital on out-patient basis and the wound healed leaving behind the scar.

FNAC suggested inflammatory pathology, possibly an infected epithelial cyst. X-ray showed an opacity below the right zygoma (Fig. 1). Surgery was done under general anaesthesia. Incision was given over the swelling and a splinter was found within a tense cystic fibrous capsule. Wound was closed and healing was uneventful.

\section{Case 2}

A 7 year old male patient presented to the OPD with painful trismus and small swelling over the left cheek for past one month. Mouth opening of only about $1.5 \mathrm{~cm}$ was possible, with painful limitation. Parents informed that the problem had an abrupt onset after he hurt himself following a fall while brushing his teeth. Subsequently he was treated by a local physician, a surgeon and a dentist who offered him different types of oral antibiotics and gargle after X-rays failed to prove any pathology. The condition did not improve by these therapies.

On examination, an ulcerated depression with oedema was found anterior to left anterior tonsillar pillar, with a pus point. It was intensely tender on palpation with a tongue

1 - Department of ENT, Medical College, Kolkata

2 - Department of ENT, IPGMER, Kolkata

\section{Corresponding author:}

Dr Bijan Kumar Adhikary

email: drbadhikary@gmail.com 


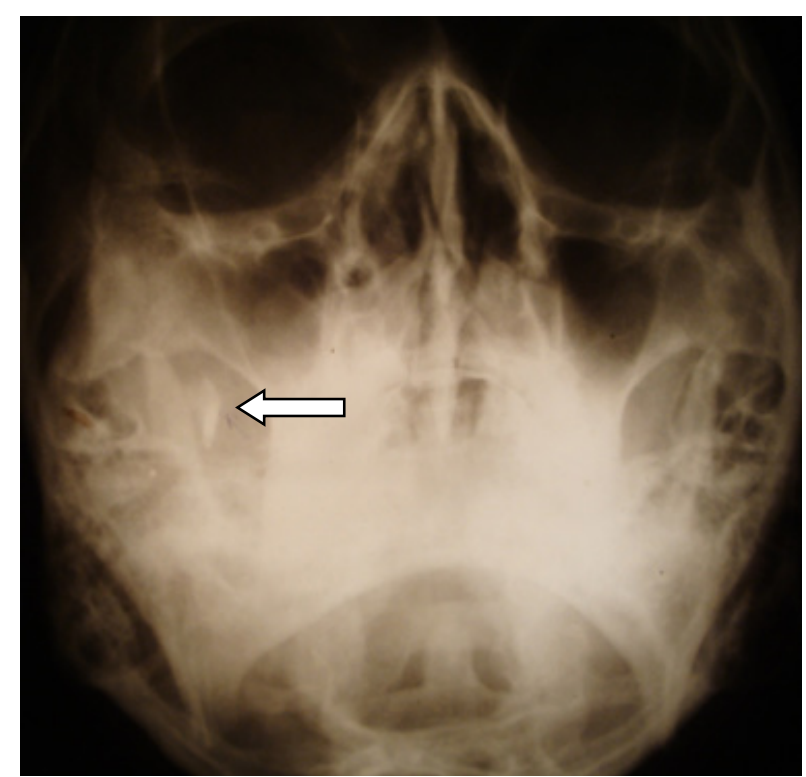

Fig. 1 White arrow showing the splinter

depressor. CT scan showed the presence of the head of a toothbrush between the medial and lateral pterygoids (Fig. 2).

Exploration was done under general anaesthesia. Plastic head of toothbrush was gripped with curved artery forceps after negotiating it through the ulcerated depression and delivered out. Finger palpation was done to confirm that there was no bristle left. Post-operative mucosal healing was adequate. Repeat CT scan was done to confirm that no bristle was left behind. There was only gas shadow at the site of impaction. Trismus gradually improved over a period of two months.

\section{Case 3}

A 6 year old male patient presented with painful swelling in the left side of the neck of ten days duration. There was also a history of painful mouth opening. Parents gave the history of an accidental fall while brushing his teeth. He was treated in a subdivisional hospital, but symptoms did not improve. Without any x-ray, he was treated by different types of antibiotics and analgesics. The patient did not fully recover from his symptoms.

On examination, a tender swelling was noted in the left submandibular region. An inflamed and indurated area was noted in the left anterior pillar of the tonsil.

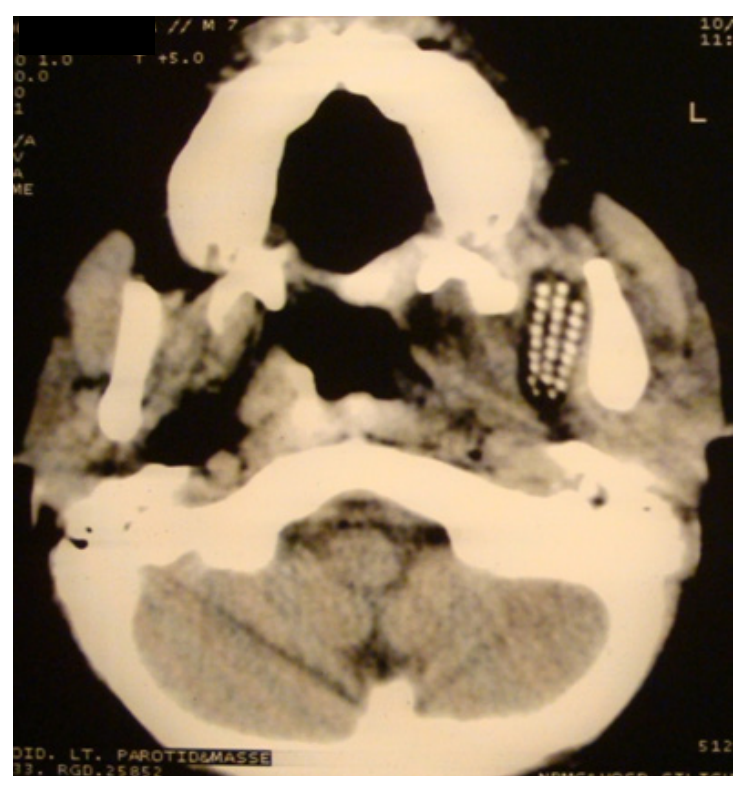

Fig. 2 CT Scan showing the head of the toothbrush

On digital X-ray, a brush like radio-opaque shadow was seen. CT scan was done to confirm the finding. A radio-opaque shadow was seen in the left submandibular triangle (Fig. 3). Exploration was done under general

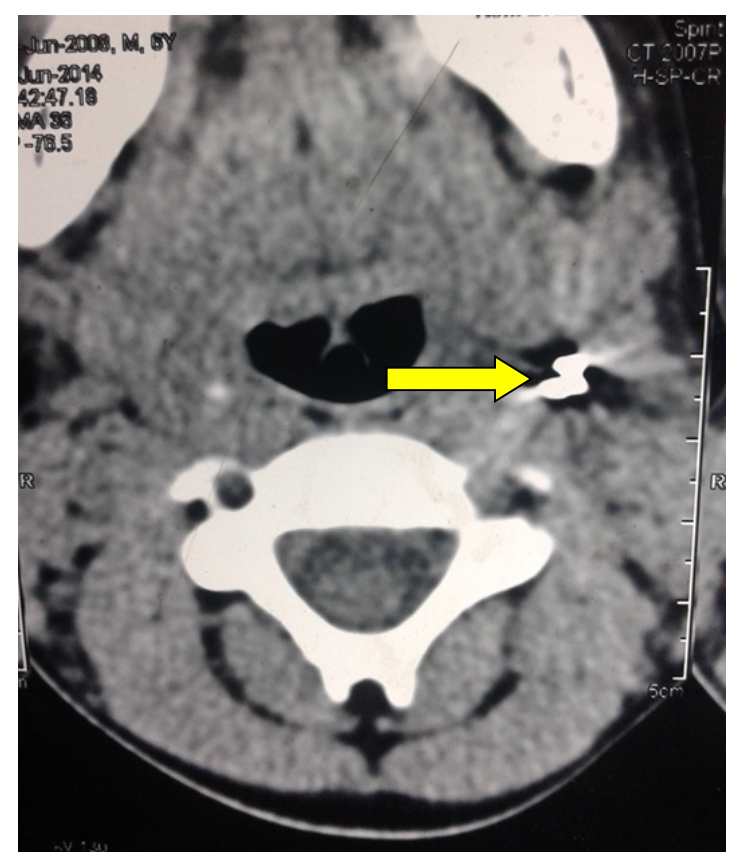

Fig. 3 CT Scan showing the broken toothbrush (Yellow arrow) 
anaesthesia. A red coloured brush was seen posterior to the tonsil. Repeated pulling by artery forceps failed to disimpact the brush. The brush head was removed by giving an incision between uvula and anterior pillar, which was closed with Vicryl ${ }^{\circledR}$ sutures. Pain and other symptoms were subsided within one week.

\section{Discussion}

The three cases presented here have the similarity that all were penetrating foreign bodies that remained within the body undiagnosed for some time before being located by proper radiological assessment. Straight X-ray is the preferred investigation to confirm the presence of a foreign body in the aerodigestive tract and to diagnose the level of its impaction. Endoscopies and examination under microscope are the preferred methods to diagnose and retrieve foreign bodies in ENT.

Computed tomography is required in cases of respiratory passage foreign bodies to learn about the site of lodgment and the effect on the respiratory system before they are removed endoscopically.

In the three cases that we present, there was no history from the patient regarding any foreign body. The foreign bodies were missed even by treating doctors in their subsequent medical consultations. In the case of retained splinter, the radiopaque shadow was missed in one plate as an improper view caused overlap of the foreign body with natural opacity of the facial bones. It was documented by X-ray skull occipitomental and lateral views.

The incidence of gunshot injuries and splinter injuries in the head and neck region have increased during the past decade, but are not as common as they are in other areas. It may cause poisoning, fistula formation, recurrent infection or secondary hemorrhage, and its extraction is necessary even in absence of clinical symptoms. However, hisory of bullet injury is there in medical literature where the foreign bodies remained asymptomatic throughout life.

Our patient had the splinter for 13 long years inside the body without any symptom. Due to some unknown reason, there developed an infection with in the fibrous capsule ensheathing the foreign body resulting in delayed presentation as an infected cyst.
In the second case, the diagnosis was missed as the father of the child insisted on the trauma from toothbrush to be the cause of the pain and trismus in the child. Incidences of impaction of toothbrush in the oral cavity and parapharyngeal space are present in the medical literature. Incidents of tooth brush swallowing are also there., ${ }^{2,3}$ But it is rare for such foreign bodies to present with no definite history of penetration. Immediate airway compromise is a threat in penetrating oropharyngeal injuries. The severity of injury is not always apparent. A foreign body can cause poisoning, fistula formation ${ }^{4}$ recurrent infection or secondary hemorrhage. Its extraction is recommended even in absence of clinical symptoms.

The second patient had no information about whether the toothbrush had broken or any part was missing. On clinical inspection there was only ulcer and local inflammation visible without any part of foreign body showing. Finger palpation was not possible due to trismus, and toothbrush being plastic was not radiopaque. It was missed on X-ray. It was only $\mathrm{CT}$ scan that could document for the first time the presence of the head of the toothbrush and it was removed under general anaesthesia. Knowledge of the anatomy of the pharynx is essential in dealing with foreign bodies in this region. ${ }^{5}$ There is incidence of development of pseudo aneurysm of the internal carotid artery after an infant fell on her face with spoon in mouth. ${ }^{6}$ Care must be taken to ensure that a missing piece of foreign body does not remain lodged ${ }^{2}$ which can be confirmed by finger palpation at time of surgery and a CT scan may be advised subsequently, if necessary. Trismus takes about two months to improve.

In the third case, parents gave a history of fall with brush in the oral cavity. But the diagnosis could not be established due to lack of X-ray and other radiological investigations. Tender and swollen areas were noted in the left submandibular region on clinical examination. Simple X-ray could diagnose the foreign body in the submandibular region. For better localization, CT scan can be done. Incision may be required in some cases to prevent injury to the vital structures. 


\section{Conclusion}

Foreign bodies can remain in the body undiagnosed for a long duration. Foreign bodies may be missed by qualified doctors if not investigated adequately. Adequate radiology is the best procedure to diagnose foreign bodies. Foreign bodies, even if asymptomatic, must be removed to prevent recurrent infection or other complications.

\section{References}

1. S Sarkar, A Roychoudhury, B K Roychoudhury. Foreign bodies in ENT in a teaching hospital in Eastern India, Indian $\mathrm{J}$ Otolaryngol Head Neck Surg 2010; 62:118-20.

2. Kirk AD, Bowers BA, Moylan JA, Meyers WC. Toothbrush Swallowing. Arch Surg. 1988;123:382-4

3. Faust J, Schreiner O. A Swallowed tooth brush. Lancet 2001; 357:1012

4. M Isa Kara, H B Polat, Sinan AY. Penetrated Shotgun Pellets: A Case ReportEuropean Journal of Dentistry 2008; 2:59-62.

5. James J. Grochowski, Brian Hynes: A toddler with a pharyngeal foreign body Canadian Family Physician 2008; 54:1695-6.

6. Chambers N, Hampson- Evans D, Murdoch L. Traumatic Aneurysm of the internal carotid artery in an infant: a surprise diagnosis. Pediatr Anaesth 2002; 12: 356-61 